\title{
Home Electric Energy Monitoring System: Design and Prototyping
}

\author{
João Gil Josué, João Murta Pina, and Mário Ventim Neves \\ Faculdade de Ciências e Tecnologia, Universidade Nova de Lisboa \\ Monte de Caparica, 2829-516 Caparica, Portugal \\ jgj@netcabo.pt, jmmp@fct.unl.pt, ventim@uninova.pt
}

\begin{abstract}
The energy resource management is a major concern worldwide. Energy management activities minimize environmental impacts of the energy production. Therefore, electric energy consumption monitoring has been proposed as an important process which makes immediate reductions in energy use and $\mathrm{CO}_{2}$ emissions. In recent years, advances in electronics have allowed the implementation of many technological solutions that could help to reduce energy consumption. This paper describes the design and prototyping of a home electric energy monitoring system that provides residential consumers with real time information about their electricity use. The system uses wireless communication and displays the information on a small LCD screen and on a computer.
\end{abstract}

Keywords: Electric energy monitoring, energy management, home automation.

\section{Introduction}

Electricity plays a crucial role in the economic and social development of the world and in the quality of life of its citizens and consumers [1]. However, electric energy production is mainly supplied by fossil fuels, such as oil, natural gas and coal. The dependency on limited fossil energy resources and the consequent greenhouse gases emissions (GHGs, including $\mathrm{CO}_{2}, \mathrm{CH}_{4}$ and $\mathrm{N}_{2} \mathrm{O}$ ) warned the world about the unsustainability of the current situation.

Investments in energy efficiency and renewable energy have been crucial measures of sustainable energy policies, since they are often economically beneficial, improve energy security and reduce local pollutant emissions [2].

In 2007, final electricity consumption in the residential sector in the European Union (EU) was $28 \%$ of the total [3]. This sector has been highlighted as an area which has a significant potential for improvement. Thus, residential sector energy efficiency programs can significantly reduce electricity consumption worldwide.

Consumers have an important role in the energy management activities and their actions represent an important step to minimize environmental impacts of energy production. Real-time electric energy information has a great impact in consumer's behaviors and habits. Past studies suggest that providing detailed and instantaneous feedback on household electrical demand can reduce electric energy consumption by $5-15 \%[4][5][6]$. 
Due to advances in electronics and computing, many technologic solutions are now available. These solutions are a very important tool to a sustainable future.

This work describes the design, prototyping and testing of a home electric energy monitoring system capable of measuring and displaying on a small LCD the real time information about consumer's electricity use. This system can also be connected to a computer (via USB) to record and analyze measured data.

The developed system is part of a global energy monitoring system (electricity, gas and water) that provides real time energy use information to increase energy efficiency. Nevertheless, in this paper, only the electrical part is described, as this is able to work independently.

\section{Contribution to Sustainability}

Nowadays, the most common type of household electricity meter is the electromechanical induction meter. However, with this type of meter consumers have no means to judge electricity use other than their monthly utility bill. Therefore, it is difficult to know the necessary measures to improve the home's energy efficiency. The proposed system aims to contribute to sustainability in the way that it readily provides insight as to how and where the electric energy is being use. Since it provides real-time information on household electrical demand and records historical measured data for future analysis, this system potentiates electric energy efficient use, leading to significant environmental, political and economic benefits.

\section{Home Electric Energy Monitoring Systems}

Today, there are many systems available to monitoring household electricity use. Smart meters can be used to replace traditional electromechanical meters and provide both the supplier and the consumer with a better control of the electricity use. The smart meter can also be a part of energy management infrastructures, such as smart grids.

However, in a consumer's point of view it is beneficial to use a low-cost, user friendly and flexible monitoring system. This type of system, which is exclusively developed to help consumers manage and reduce their electricity use, doesn't replace the traditional meter and has the advantage of being portable. Depending on their sophistication, these monitoring systems can also communicate with a computer or a cell phone, send data throughout the house using wireless communications or connect to the internet allowing remote monitoring.

In recent years, several home electric energy monitoring systems have emerged on the market. Some systems can be plugged into the wall outlet to measure appliance's consumptions. Furthermore, other approaches measure total household electricity use through appropriate sensors installed into a home's switchboard circuits.

\section{Proposed Monitoring System}

The developed system consists of two electronic devices: data acquisition device and data display device, presented in figure 1. The data acquisition device measures 
power and energy consumed by loads and the data display device displays measured data on a small LCD screen and sends results to the computer.

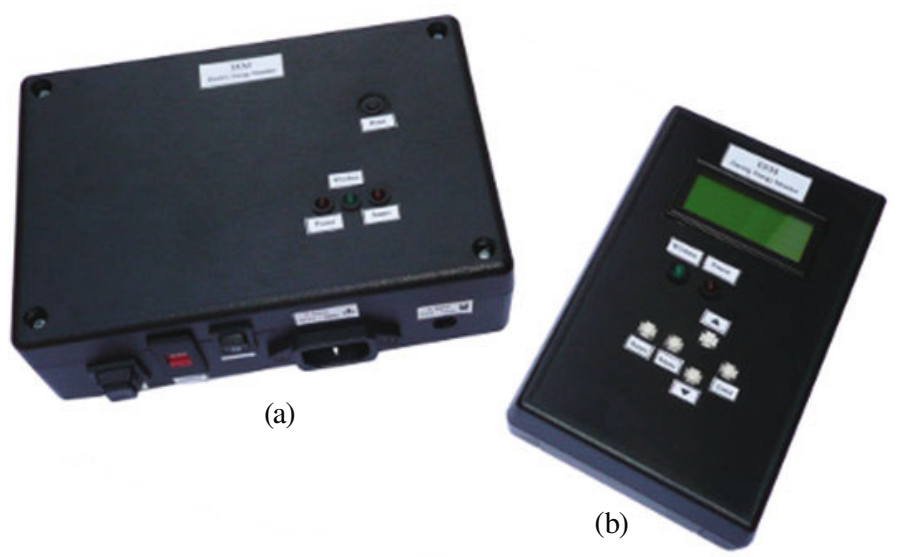

Fig. 1. Developed monitoring system's devices a) Data acquisition device b) Data display device

The main features of this monitoring system are: wireless communication between acquisition and display devices, monitoring capability at the appliance and switchboard circuit's level, average hourly energy use and electricity cost information display, and data recording on the computer.

Wireless communication between devices ensures greater flexibility and system's ease of use. The system's ability to monitor both appliance level and switchboard circuit's level informs the consumer about the balance of each appliance or circuit load. The knowledge of average hourly energy use and electricity cost provides important information that motivates changes to consumer's behavior. A computer connection is available to record measured data and to use it in many computer applications, such as, daily charting energy use data.

\subsection{General Architecture}

The data acquisition device diagram block is represented in figure 2 . This device consists of five major blocks: power integrated circuit (IC), microcontroller, wireless transceiver, signal conditioning and power supply.

The data acquisition device measures line voltage and current signals through appropriate sensors. These analog signals are then conditioned and used by a power IC, which measure RMS voltage, RMS current, power factor and active power. This information is transmitted to a microcontroller that computes the energy consumed by a load and communicates with a wireless transceiver. The transceiver is responsible for sending the measured data to the data display device and for receiving commands from the user. The microcontroller can also communicate with status LEDs and with a reset button. The device power supply provides 5V DC from $230 \mathrm{~V}$ AC line. 


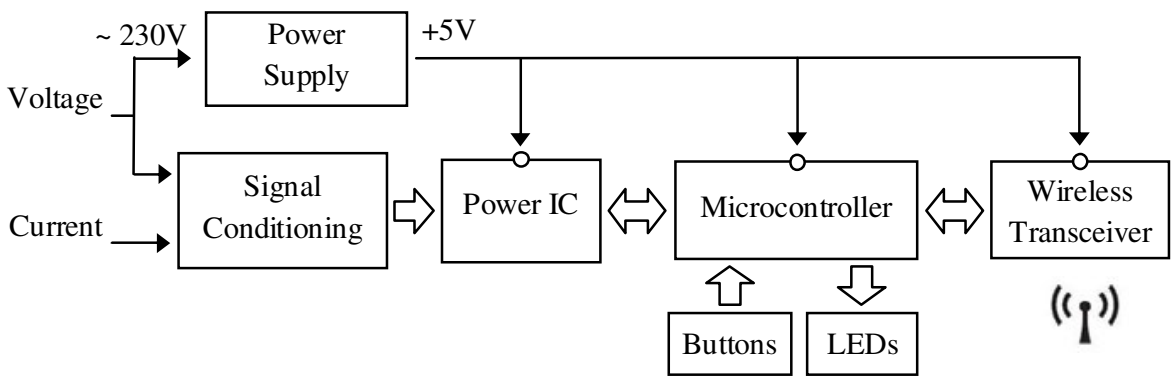

Fig. 2. Data acquisition device diagram block

Figure 3 presents data display device diagram block. This device consists of four major blocks: microcontroller, wireless transceiver, LCD and power supply.

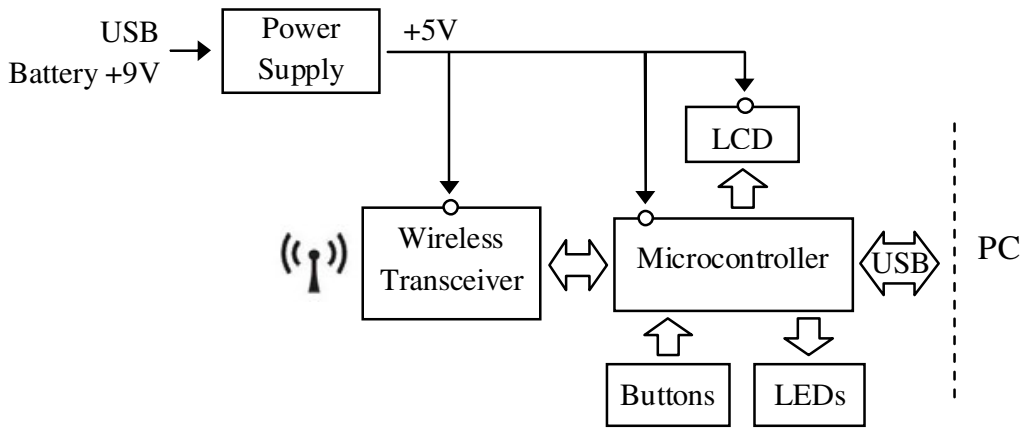

Fig. 3. Data display device diagram block

Data display device receives measured data from the data acquisition device through a wireless transceiver. The transceiver sends the received information to a microcontroller. This microcontroller is responsible for several operations, including LCD data driving, communication with a computer via USB, LEDs and buttons control and electricity cost calculation. The device buttons allow the user to select and perform many functions, such as data measurement initialization, power IC calibration and electricity tariff definition. This device is supplied by a $9 \mathrm{~V} \mathrm{DC}$ battery or $5 \mathrm{~V}$ DC USB interface.

\subsection{Hardware}

Power IC. The power measurement is performed by an analog integrated circuit design for residential single-phase, the CS5463 from Cirrus Logic. This IC focuses all the calculation complexity on a single circuit and is a highly accurate and a costeffective solution. It is equipped with a computation engine that calculates RMS voltage, RMS current, active and reactive power and power factor within an accuracy of $0.1 \%$. For communication with a microcontroller, the IC features a bi-directional serial interface which is SPI compatible. 
Wireless Transceiver. Data communication between the acquisition and display devices is achieved by two wireless transceivers ER400TRS, developed by EasyRadio. These transceivers combine high performance low power RF, operate on $433 \mathrm{MHz}$ frequency band, have a range of up to 250 meters line of sight, and provide serial interface for connection to UART devices.

LCD. The energy use information is displayed on a small LCD panel, developed by Batron, with 20 characters and 4 lines.

Microcontrollers. To control operations of many electronic components and endow the system with some intelligence, each device has an 8 bit microcontroller from Microchip. Data acquisition device is based on a PIC18F2420, which supports SPI and UART communication, while data display device uses a PIC18F2550 that provides a USB interface for communication with a computer.

Current Sensor. Current signal is read by a split core current transformer (CT) developed by CR Magnetics, model CR3110. This type of sensor enables the electricity monitoring at switchboard circuit's level, because there is no need to interrupt the circuit. CR3110 was designed to preserve linearity over a large current range, up to 65 A. Therefore, a burden resistor of $10 \Omega$ was used for this system. This CT introduces a phase shift between the primary and secondary current. In this design, the phase shift was partially removed by the CS5463 throughout phase compensation.

Signal Conditioning. Voltage and current signals are conditioned by resistive networks and low pass filters to adjust their levels before being applied to the CS5463.

Power Supply. A transformer isolated power supply provides power to the data acquisition device. The AC from the center tapped secondary is full wave rectified, filtered and provided to the $5 \mathrm{~V}$ regulator. The $5 \mathrm{~V}$ loads are the CS5463, the PIC18F2420, the ER400TRS and the LEDs. Data acquisition device draws less than $47 \mathrm{~mA}$.

Data display device power supply comprises a $9 \mathrm{~V}$ battery and a $5 \mathrm{~V}$ voltage regulator to supply the PIC18F2550, the ER400TRS, the LCD and the LEDs. These components draw about $63 \mathrm{~mA}$.

Protections. To protect data acquisition device against possible surges, overloads and electromagnetic noise, a varistor, a circuit breaker and a line filter was used.

\subsection{Firmware}

The PIC18 microcontroller's firmware was developed in $\mathrm{C}$ and compiled with MPLAB C18 from Microchip. Each microcontroller (PIC18F2440 and PIC18F2550) need to perform many events. To provide real time response to these events, the microcontroller's interrupts and the interrupt services routines were used. A set of routines were developed for the two microcontrollers, which implement many real time events. The most important routines developed are listed below in table 1 and table 2 . 
Table 1. Data acquisition device PIC18F2420 routines

\begin{tabular}{|c|l|}
\hline Routine & \multicolumn{1}{c|}{ Function } \\
\hline UART receiver ISR & - Detect messages sent by the data display device \\
\hline Timer 1 ISR & $\begin{array}{l}\text { - Read CS5463 registers which have measured energy data; } \\
\text { - Calculate energy consumed; } \\
\text { - Send data to the data display device; }\end{array}$ \\
\hline CS5463 calibration & - Eliminate CS5463 input channels offset \\
\hline
\end{tabular}

Table 2. Data display device PIC18F2550 routines

\begin{tabular}{|c|l|}
\hline Routine & \multicolumn{1}{c|}{ Function } \\
\hline UART receiver ISR & - Detect messages sent by the data acquisition device \\
\hline Timer 0 ISR & - Update data displayed in the LCD \\
\hline USB interface & - Control data transmission through USB interface \\
\hline Port B & - Detect an input change in buttons corresponding pins \\
\hline
\end{tabular}

\subsection{Software}

A software application, based in C\#, was also developed for the computer. This application communicates with the data display device through a USB interface and enables receiving and storing the monitoring system measured data. This program also provides daily charting energy use data giving a very useful visual tool to the user. Figure 4 presents some screens from the developed software application.

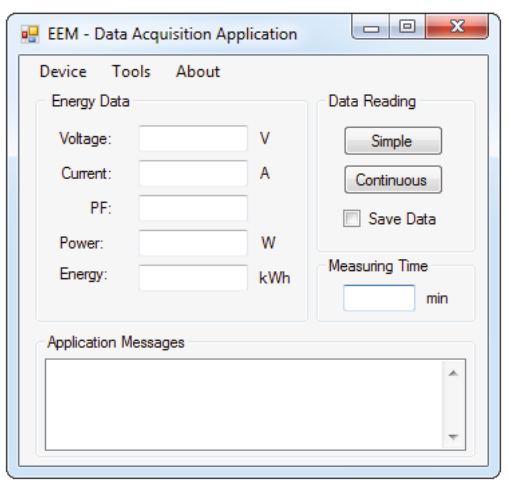

(a)

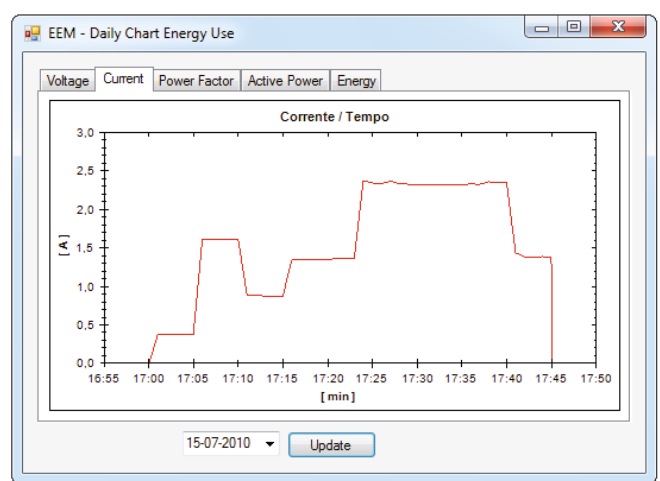

(b)

Fig. 4. a) Software application interface b) Daily chart energy use window 


\subsection{Prototyping}

The developed system prototyping consists of three main parts: PCBs (printed circuit board) design and production, electronic components PCB welding and components boxes assembly. The prototype production cost was €280.82. To enable the appliance level monitoring, a module to be plugged into the wall outlet was also built. This module reads the line voltage signal and provides a wire for plugging the current sensor.

\section{Results}

In order to ensure a suitable data accuracy measurement, the proposed system was calibrated through a three-step process: CS5463 input channels offset elimination, phase shift compensation and scaling factors adjustment. To validate the system and verify its accuracy, several tests were carried out. These tests showed that data acquisition device has a measurement error of less than $1 \%$ for loads greater than $0,5 \mathrm{~A}$.

Finally, several functional tests were performed, to validate the system's behavior in real situations. The system was tested on both appliance level and switchboard circuit's level. It was possible to validate all the system's devices and components, which have a proper operation and integration.

\section{Conclusions and Further Work}

This paper describes the design and prototyping of a home electric energy monitoring system, which has been successfully completed. The experimental results have demonstrated that the proposed system is accordance with the design's specifications. Besides its original goal of integrating a global energy monitoring system, the developed system can also be used in energy auditing and energy advising processes.

Envisaged future work consists in new current sensor adding in order to double the data acquisition capacity, PCB's and component's size reduction by using SMT technology and internal memory installation to record measured data directly in the system.

\section{References}

1. Commission of the European Communities: Europe's current and future energy position Demand - resources - investments. Second Strategic Energy Review. p. 38. Brussels (2007)

2. Solomon, S., Qin, D., Manning, M., Chen, Z., Marquis, M., Averyt, K.B., Tignor, M., Miller, H.L. (eds.): Climate Change 2007: The Physical Science Basis. Contribution of Working Group I to the Fourth Assessment Report of the Intergovernmental Panel on Climate Change. Cambridge University Press, Cambridge (2007)

3. Joint Research Centre (JRC): EU energy efficiency measures contribute to stabilize electricity consumption - drop in domestic use. Brussels (2009) 
4. Mountain, D.: The impact of Real-Time Feedback on Residential Electricity Consumption: The Hydro One Pilot. Mountain Economic Consulting and Associates Inc., Ontario (2006)

5. Darby, S.: The Effectiveness of Feedback on Energy Consumption. Environmental Change Institute of University of Oxford, UK (2006)

6. Parker, D.S., Hoak, D., Cummings, J.: Pilot Evaluation of Energy Savings from Residential Energy Demand Feedback Devices. Final Report by the Florida Solar Energy Center to the U.S. Department of Energy (2008) 